\title{
EXACTLY SOLUBLE DILUTED RANDOM ONE-DIMENSIONAL LATTICES
}

\author{
Th.M. NIEUWENHUIZEN \\ Institute for Theoretical Physics, Princetonplein 5, P.O. Box. 80.006, 3508 TA Utrecht, The Netherlands
}

Received 25 January 1984

Revised manuscript received 1 May 1984

Exact solutions for the characteristic function, which determines the density of states and inverse localization length, and one-particle Green function are presented for a class of lattice models with diluted randomness. (Examples are: harmonic, electronic, relaxation and $X-Y$ spin systems.) With probability $1-p$ the random variables assume a constant value; with probability $p$ they have values taken from an exponential distribution (diagonal disorder) or from a power-law distribution (off-diagonal disorder).

In this letter we consider a class of one-dimensional random models, e.g. tight-binding electron models, harmonic chains, random relaxation models, random alloys, $X-Y$ models. For these systems the equation of motion may be written in the form

$\left(2 \cosh \mu+2 \eta x_{j} \sinh \mu\right) u_{j}=u_{j+1}+u_{j-1}$,

where $x_{j}$ is a parameter connected to the random site energy, mass, jump-rate, delta-potential strength, magnetic field. For example, in the tight-binding model with random site energies $\epsilon_{j}=\epsilon+\delta x_{j}$ one has $2 \mathrm{X}$ $\cosh \mu=E-\epsilon, 2 \eta \sinh \mu=-\delta$; in the harmonic chain with random masses $m_{j}=m+M x_{j}$ one has $2 \cosh \mu=2$ $+m \xi, 2 \eta \sinh \mu=M \xi$, where $\xi=-\omega^{2}$. In such systems a "characteristic function" can be defined $[1,2]$ which determines the density of states and the inverse localization length of the eigenfunctions $[3,4]$. Of special interest are models where this characteristic function can be solved exactly. For the random alloy such solu. tions were discussed before [5] for exponential distributions of the $x_{n}$; for the tight-binding and $X-Y$ models, relaxation models and the harmonic chain with random masses, apart from this function, also the oneparticle Green function was given in explicit form [6].

Exactly solved models with configurational disorder, where only a fraction $p(0 \leqslant p \leqslant 1)$ of the lattice sites contains a random parameter, are not known to us, except for a tight-binding model where the signs of the hopping energies are random [6]. They might be of interest, e.g., in diffusion controlled reactions on lattices or in relaxation models on lattices with randomly distributed impurities.

It is the purpose of this letter to show that the abovementioned exact solutions for the characteristic function and Green function can be generalized to diluted cases. We shall take as probability distribution of the $x_{j}$

$$
\begin{aligned}
\rho\left(x_{j}\right) & =p \exp \left(-x_{j}\right)+r \delta\left(x_{j}\right) & & \left(x_{j} \geqslant 0\right), \\
& =0 & & \left(x_{j}<0\right),
\end{aligned}
$$

where $r=1-p$.

For complex values of the energy or frequency the characteristic function may be defined as [2]

$\Omega=\lim _{N \rightarrow \infty}\left\langle N^{-1} \log u_{N}\right\rangle=\lim _{N \rightarrow \infty}\left\langle\log \left(u_{N} / U_{N-1}\right)\right\rangle$,

where $u_{N}$ is the solution of (1) with boundary conditions $u_{0}=0, u_{1}=1$. In the tight-binding model it has along the real $E$-axis the decomposition into real and imaginary parts

$\Omega(E+\mathrm{i} 0)=\gamma(E) \pm \mathrm{i} \pi[1-H(E)]$.

In the harmonic chain with random masses the decomposition of $\Omega(\xi)$ along the negative real $\xi$-axis is 
$\Omega\left(\xi=-\omega^{2} \pm \mathrm{i} 0\right)=\gamma\left(\omega^{2}\right) \pm \mathrm{i} \pi H\left(\omega^{2}\right)$.

In $(4 a, b)$ the quantity $\gamma$ is the exponential growth rate or inverse localisation length of the eigenfunctions $[3,4]$ and $H$ is the integrated density of states per lattice site. For the calculation of $\Omega$ we have introduced in a previous paper [2] the function

$D(u)=\lim _{N \rightarrow \infty}\left\langle\log \left(u_{N} / u_{N-1}-1 / u\right)\right\rangle$.

The function $\Omega$ is given by $D(\infty)$. Using eq. (1) one derives the following equation for $D(u)$

$D(u)=\int \rho(x) \mathrm{d} x[D(v)+\log v]-\Omega$

$$
(v=2 \cosh \mu+2 \eta x \sinh \mu-1 / u) .
$$

The method of constructing the solution for $\Omega$ for the non-diluted case $p=1$ was developed in ref. [5]. We follow the lines of this paper and perform differentiation and partial integration to eq. (5) in order to remove the integration over $\rho(x)$

$-2 \eta \sinh \mu u^{2} \partial_{u}\left[D(u)-r D\left(u^{\prime}\right)-r \log u^{\prime}\right]$

$$
=-D(u)+D\left(u^{\prime}\right)+\log u^{\prime}-\Omega,
$$

where $u^{\prime}=2 \cosh \mu-1 / u$. This equation takes the form of a differential-difference equation for

$G(z)=D(u(z))+\log \left(1-\mathrm{e}^{-z}\right) / 2 \sinh \mu$,

where $u(z)=\left(\mathrm{e}^{z}-1\right) /\left(\mathrm{e}^{z-\mu}-\mathrm{e}^{\mu}\right)$. Substituting

$G(z)=\sum_{k=1}^{\infty} c_{k}\left(1-\mathrm{e}^{-k z}\right) / k$

the equations for the coefficients $c_{k}$ may be written

$\widetilde{c}_{k+1}+\widetilde{c}_{k-1}=\left(2+\frac{1}{\eta k} \frac{1-\mathrm{e}^{-2 k \mu}}{1-r \mathrm{e}^{-2 k \mu}}\right) \tilde{c}_{k} \equiv \rho_{k} \tilde{c}_{k}$,

$\Omega=\mu+p \eta\left(1-\widetilde{c}_{1}\right)$,

where $\tilde{c}_{k}=\left(1-r \mathrm{e}^{-2 k \mu}\right) c_{k} / p$. The boundary conditions $\widetilde{c}_{0}=c_{0} \equiv 1$ and $\lim _{k \rightarrow \infty} c_{k}=0$ fix the solution of the recurrence relation and hence determine $\Omega$. This function may be written in explicit form by dividing (7) and $\widetilde{c}_{k}$ and iterating this equation. The result is

$\Omega=\mu+p \eta\left(1-\frac{1}{\rho_{1}-} \frac{1}{\rho_{2}-} \frac{1}{\rho_{3}-} \cdots\right)$.

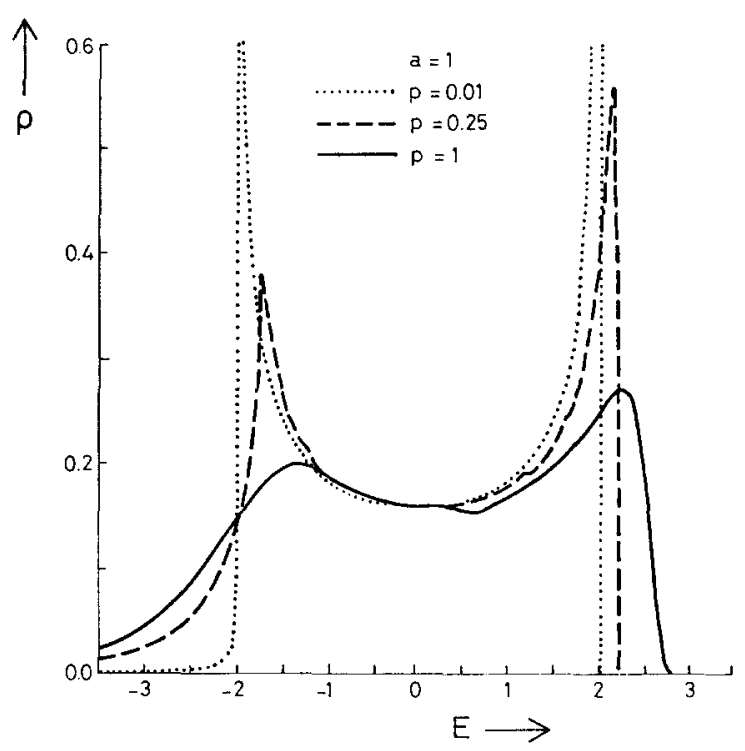

Fig. 1. Density of states $\rho(E)$ as function of the energy $E$ in a tightbinding model for several values of $p$, the fraction of sites with random, exponentially distributed site energies.

This expression in the form of a continued fraction is slightly more complicated than the non-diluted case $p=1$, for which situation graphs of density of states and $\gamma$ were presented $[5,6]$.

In figs. 1 and 2 we have plotted the density of states $\rho(E)=\mathrm{d} H(E) / \mathrm{d} E$ and inverse localization

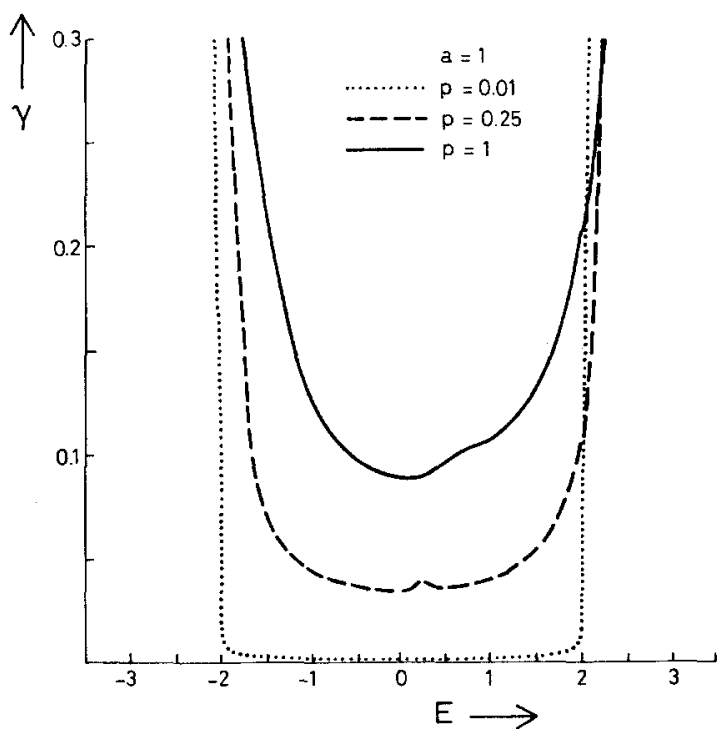

Fig. 2. Same as fig. 1, for the inverse localization length $\gamma(E)$. 
length $\gamma(E)$ as function of the energy for a tight-binding electron model with $\epsilon_{j}=-a x_{j}+p a$, implying $\left\langle\epsilon_{j}\right\rangle$ $=0$. We have taken $a=1$ and the values $p=0.01, p$ $=0.25$ and $p=1$. The first case is close to the ordered situation $\epsilon_{j}=0$, where $\rho=0$ for $|E|>2$ and has square root singularities near $E= \pm 2 ; \gamma=0$ for $|E|<2$ (delocalized states). For $p>0 \gamma$ is positive everywhere (localized states). In the case $p=1$ the disorder is strongest. The density of states is zero for $E>2+p a$. For $E \rightarrow-\infty$ it goes like $a p \mathrm{e}^{E / a}$, related to the fact that such states are located approximately at one site $j_{0}$, which has a large negative value of $\epsilon_{j_{0}}$, occurring with probability ap $\exp \left(\epsilon_{j_{0}} / a\right)$.

In the same manner the Fourier component $\mathcal{g}(q)$ of the one-particle Green function

$\left(2 \cosh \mu \delta_{j j^{\prime}}+2 \eta x_{j} \sinh \mu \delta_{i j^{\prime}}-\delta_{j, j^{\prime}+1}-\delta_{j, j^{\prime}-1}\right)^{-1}$ can be calculated for complex values of the energy or frequency. It can also be expressed in terms of $u_{n}$ and a set of integral equations similar to eq. (5) can be derived [6]. Since the derivation is long and technical we only quote the result: $g(q)=-R_{2}(0)$, where $R_{2}(u)$ and $R_{1}(u)$ have to be solved from the equations $u \mathrm{e}^{-\mathrm{i} q} R_{1}(u)=\int \rho(x) \mathrm{d} x\left[R_{1}(v)+R_{0}(v)\right]$, $R_{2}(u)=\int \rho(x) \mathrm{d} x\left[R_{2}(v)+R_{1}(v)+\bar{R}_{1}(v)+R_{0}(v)\right]$

$$
+R_{2}(0) \text {. }
$$

Here $R_{0}(u)=D^{\prime}(u)+1 / u, v$ was given in $(5 \mathrm{~b})$ and $\bar{R}_{1}(v$, $q)=R_{1}(v,-q)$. We follow the method described above and define, like in ref. [6],

$$
\begin{aligned}
& R_{1}(u(z))=\frac{\mathrm{e}^{z-\mu}-\mathrm{e}^{\mu}}{2 \sinh \mu} \sum_{k=1}^{\infty} \mathrm{d}_{k} \mathrm{e}^{-k z}, \\
& R_{2}(u(z)) \\
& \quad=(2 \sinh \mu)^{-1} \sum_{k=1}^{\infty} e_{k}\left(1-\mathrm{e}^{-k z}\right) / k-g(q),
\end{aligned}
$$

together with $d_{0}=e_{0}=0$ and write $\bar{d}_{k}$ for $d_{k}(-q)$. The equations for the coefficients $d_{k}$ and $e_{k}$ may be cast in the form

$$
\begin{aligned}
& (k-1) \tilde{d}_{k-1}+k \tilde{d}_{k+1} \\
& -\left(2 k-1+\frac{1-\mathrm{e}^{\mathrm{i} q+\mu-2 k \mu}}{\eta\left(1-r \mathrm{e}^{\mathrm{i} q+\mu-2 k \mu}\right)}\right) \tilde{d}_{k}=p \sigma_{k} / \eta,
\end{aligned}
$$

$$
\begin{aligned}
& \tilde{e}_{k-1}+\tilde{e}_{k+1}-\rho_{k} \tilde{e}_{k}=p \tau_{k} / \eta\left(1-r e^{-2 k \mu}\right), \\
& g(q)=(2 \sinh \mu)^{-1}\left(-1+\tau_{0}-\eta \tilde{e}_{1}\right),
\end{aligned}
$$

where

$$
\begin{aligned}
\sigma_{k} & =\mathrm{e}^{\mathrm{i} q-2 k \mu}\left(c_{k}-c_{k-1} \mathrm{e}^{2 \mu}\right) /\left(1-r \mathrm{e}^{\mathrm{i} q+\mu-2 k \mu}\right), \\
\tau_{k} & =\mathrm{e}^{\mu-2 k \mu}\left[\mathrm{e}^{-2 \mu}\left(d_{k+1}+\bar{d}_{k+1}\right)-d_{k}-\bar{d}_{k}\right] \\
& +\mathrm{e}^{-2 k \mu}\left(-c_{k+1} \mathrm{e}^{-2 \mu}+2 c_{k}-c_{k-1} \mathrm{e}^{2 \mu}\right), \\
\tau_{0} & =\mathrm{e}^{-\mu}\left(d_{1}+\bar{d}_{1}\right)-c_{1} \mathrm{e}^{-2 \mu}+2,
\end{aligned}
$$

and

$\tilde{d}_{k}=\left(1-r \mathrm{e}^{\mathrm{i} q+\mu-2 k \mu}\right)\left(d_{k}+r \sigma_{k}\right)$,

$\tilde{e}_{k}=\left(1-r \mathrm{e}^{-2 k \mu}\right) e_{k}+r k \tau_{k}$.

Because $d_{k}$ and $e_{k} \rightarrow 0$ for $k \rightarrow \infty$ the recurrence relations for $\tilde{d}_{k}$ and $\widetilde{e}_{k}$ can be inverted. Multiplying (12b) with $\tilde{c}_{k}$ and summing we obtain $\tilde{e}_{1}$. Inserting this in (12c) we find for the Green function the explicit expression

$g(q)=\left(-1+\sum_{k=0}^{\infty} c_{k} \tau_{k}\right)(2 \sinh \mu)^{-1}$

Note that, in terms of the coefficients $c_{k}$ and $d_{k}$, this expressidn has the same form as in the non-diluted case $p=1$. This should be because $\mathcal{G}(q)$ can also be calculated from an integral which contains $R_{0}(u)$ and $R_{1}(u)$ but not the distribution function $\rho(x)$ [6].

The solution for $\Omega$ in the tight-binding model can also be considered in the limit where the lattice constant $a$ vanishes. Inserting $E=2+\kappa^{2} a^{2}, \epsilon_{j}=-2 a V x_{j}$ and $p=a c$ in (1) we obtain for $\psi(y=j a)=u_{j}$ in the limit $a \downarrow 0$, while the other parameters are fixed,

$$
-\frac{1}{2} \psi^{\prime \prime}(y)+\sum_{i} V x_{i} \delta\left(y-y_{i}\right) \psi(y)=-\frac{1}{2} k^{2} \psi(y) .
$$

This equation describes spinless electrons in a liquid alloy with exponentially distributed delta-potential strengths (the distribution function of the $x_{i}$ is $\left.\exp \left(-x_{i}\right)\right)$. The distribution of the distances between the potentials, $\Delta_{i}=y_{i+1}-y_{i}$, is also exponential: $c \exp \left(-c \Delta_{i}\right)$, where $c$ is the concentration. In order to obtain the density of states per unit length, a factor $1 / a$ has to be added to (3) before the limit $a \downarrow 0$ is taken. The resulting expression for $\Omega$ is 


$$
\begin{aligned}
\Omega & =\kappa+\frac{c V}{\kappa}\left(1-\frac{1}{\rho_{1}-} \frac{1}{\rho_{2}-} \frac{1}{\rho_{3}-} \cdots\right) \\
& =-2 \kappa \frac{W_{\alpha, 1 / 2}^{\prime}(\kappa, V)}{W_{\alpha, 1 / 2}(\kappa, V)},
\end{aligned}
$$

where $\rho_{k}=2+\kappa(V k+c V / 2 \kappa)^{-1}, \alpha=-c / 2 k$ and $W$ is a Whittaker function. A similar formula was derived before, for slightly more general distributions of the parameters $x_{j}[5]$.

Apart from the models mentioned above and related ones, also tight-binding models with off-diagonal elements equal to $V$ (probability $r$ ) or assuming values between 0 and $V$ according to a power-law distribution can be solved in the same spirit. For the non-diluted variant of this model (and the equivalent $X-Y$ model with random exchange constants) it was already shown in ref. [6] that similar equations for the coefficients $c_{k}, d_{k}, e_{k}$ can be derived; the diluted variant can be treaten in a similar fashion as described above.

Next we consider, instead of (4), the more general distributions

$$
\begin{aligned}
\rho\left(x_{j}\right) & =p P_{+}\left(x_{j}\right) \exp \left(-n_{+} x_{j}\right)+r \delta\left(x_{j}\right) & & \left(x_{j} \geqslant 0\right), \\
& =p P_{-}\left(x_{j}\right) \exp \left(b x_{j}\right) & & \left(x_{j}<0\right),
\end{aligned}
$$

where $P_{ \pm}(x)$ are given, properly normalized polynomials of order $n_{+}-1$ and $n_{-}-1$, respectively. In this case $\left(2 n_{+}+2 n_{-}\right)$-step recursion relations for the coefficients $c_{k} ; d_{k}$ and $e_{k}$ can be derived [5]. These may be solved numerically; see ref. [6] for a harmonic chain with random masses $m_{j}=x_{j}$ where they become higher order differential equations. In the special case of gamma-distributions one has $n_{+} \equiv n, P_{-}$ $=0$ and $P_{+}(x)=(n x)^{n-1} n /(n-1)$ ! In the limit $n$ $\rightarrow \infty$ they yield the binary distribution $\rho(x)=p \delta(x$ $-1)+r \delta(x)$, for which it is known that the spectrum may have a very detailed behaviour, including infinitesimal gaps [7] and absence of a smooth density of states [8]. Obviously such a model cannot be solved exactly. If, however, the continuum limit $a \downarrow 0$ is taken, the Schrödinger equation for electrons in a liquid of point particles with electronic delta potentials is ob- tained (i.e. eq. (15) with $x_{i}=1$ ). The solution for the density of states [9] and $\Omega$ [5] may be reduced to second order differential equations. In the more general case of hard-rod liquid molecules with arbitrary but given electronic potentials (vanishing outside the rods) $\Omega$ can be solved from two-step recurrence relations for the coefficients $c_{k}$ [5]. The density of states is smooth because the (exponential) distribution of the intermolecular distances is smooth.

Finally we consider the Lloyd model [10], a tightbinding model where the $\epsilon_{j}$ have Cauchy distributions with parameter $\delta$. For arbitrary lattices the density of states and the average one-particle Green function are obtained from the ordered model $\epsilon_{j}=V$ after the replacement $V \rightarrow \mathrm{i} \Gamma$. If one would try to solve the diluted variant of this model in the same way, one would first have to solve the binary model with $\epsilon_{j}=V_{1}$ or $V_{2}$ and then make the replacement $V_{2} \rightarrow \mathrm{i} \Gamma$. This implies presumably that an exact solution cannot be obtained.

It is a pleasure to thank Martin A. Burschka for a discussion which led to the solution of the model and dr. M.H. Ernst for further discussions on the subject. This work was sponsored by the "Stichting voor Fundamenteel Onderzoek der Materie (F.O.M.)", which is supported by the "Stichting voor Zuiver Wetenschappelijk Onderzoek (Z.W.O.)".

\section{References}

[1] F.J. Dyson, Phys. Rev. 92 (1953) 1331.

[2] Th.M. Nieuwenhuizen, Physica 113A (1982) 173.

[3] H. Matsuda and K. Ishii, Supp. Prog. Theor. Phys. 45 (1970) 56.

[4] D.J. Thouless, J. Phys. C5 (1972) 77.

[5] Th.M. Nieuwenhuizen, Physica 120A (1983) 468.

[6] Th.M. Nieuwenhuizen, Physica A (1984), to be published; Thesis (State University, Utrecht, The Netherlands, 1983).

[7] J. Hori, Spectral properties of disordered chains, ed. D. ter Haar (Pergamon, Oxford, 1968).

[8] J.E. Gubernatis and P.L. Taylor, J. Phys. C4 (1971) L94.

[9] H.L. Frisch and S.P. Lloyd, Phys. Rev, 120 (1960) 1175.

[10] P. Lloyd, J. Phys. C2 (1969) 1717. 